\title{
Monitoring Leishmania infection and exposure to Phlebotomus perniciosus using minimal and non-invasive canine samples
}

Carla Maia ${ }^{1,2^{*}+}$ (D) , José Cristóvão ${ }^{1,2+}$, André Pereira ${ }^{1,2}$, Tatiana Kostalova ${ }^{3}$, Tereza Lestinova ${ }^{3}$, Petra Sumova ${ }^{3}$, Petr Volf ${ }^{3}$ and Lenea Campino ${ }^{2}$

\begin{abstract}
Background: In endemic areas of zoonotic leishmaniosis caused by L. infantum, early detection of Leishmania infection in dogs is essential to control the dissemination of the parasite to humans. The aim of this study was to evaluate the serological and/or molecular diagnostic performance of minimally and non-invasive samples (conjunctiva cells (CS) and peripheral blood (PB)) for monitoring Leishmania infection/exposure to Phlebotomus perniciosus salivary antigens in dogs at the beginning and the end of sand fly seasonal activity (May and October, respectively) and to assess associated risks factors.

Methods: A total of 208 sheltered dogs from endemic areas of leishmaniosis were screened. Leishmania DNA detection in PB on filter paper and CS was performed by nested-PCR (nPCR), while the detection of anti-Leishmania antibodies was performed using IFAT and ELISA. The exposure to P. perniciosus salivary antigens (SGH, rSPO1 and rSP03B + rSP01) was measured by ELISA.

Results: Ninety-seven (46.6\%) and 116 (55.8\%) of the 208 dogs were positive to Leishmania antibodies or DNA by at least one test at the beginning and end of the sand fly season, respectively. IFAT and ELISA presented a substantial agreement in the serodiagnosis of leishmaniosis. Discrepant PB nPCR results were obtained between sampling points. Leishmania DNA was detected in CS of 72 dogs at the end of the phlebotomine season. The presence of antibodies to the parasite measured by ELISA was significantly higher in dogs presenting clinical signs compatible with leishmaniosis at both sampling points. Phlebotomus perniciosus salivary antibodies were detected in 179 (86.1\%) and 198 (95.2\%) of the screened dogs at the beginning and end of the phlebotomine season, respectively.

Conclusions: The association between ELISA positivity and clinical signs suggests its usefulness to confirm a clinical suspicion. CS nPCR seems to be an effective and non-invasive method for assessing early exposure to the parasite. PB nPCR should not be used as the sole diagnostic tool to monitor Leishmania infection. The correlation between the levels of antibodies to P. perniciosus saliva and Leishmania antibodies suggests the use of a humoral response to sand fly salivary antigens as biomarkers of $L$. infantum infection.
\end{abstract}

Keywords: Blood, Conjunctival cells, Dog, Exposure, L. infantum, Phlebotomus pernicious, Saliva

\footnotetext{
*Correspondence: carlamaia@ihmt.unl.pt; cmaia.parasitevectors@gmail.com

${ }^{\dagger}$ Carla Maia and José Cristóvão contributed equally to this work

1 Global Health and Tropical Medicine (GHTM), Instituto de Higiene e

Medicina Tropical (IHMT), Universidade NOVA de Lisboa (UNL), Lisbon,

Portugal

Full list of author information is available at the end of the article
}

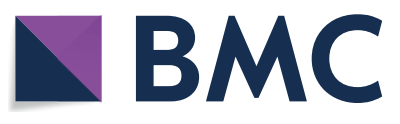

(c) The Author(s) 2020. This article is licensed under a Creative Commons Attribution 4.0 International License, which permits use, sharing, adaptation, distribution and reproduction in any medium or format, as long as you give appropriate credit to the original author(s) and the source, provide a link to the Creative Commons licence, and indicate if changes were made. The images or other third party material in this article are included in the article's Creative Commons licence, unless indicated otherwise in a credit line to the material. If material is not included in the article's Creative Commons licence and your intended use is not permitted by statutory regulation or exceeds the permitted use, you will need to obtain permission directly from the copyright holder. To view a copy of this licence, visit http://creativecommons.org/licenses/by/4.0/. The Creative Commons Public Domain Dedication waiver (http://creativecommons.org/publicdomain/zero/1.0/) applies to the data made available in this article, unless otherwise stated in a credit line to the data. 


\section{Background}

Canine leishmaniosis (CanL) caused by the protozoan Leishmania infantum is endemic in several countries of Central and South America, the Mediterranean Basin, Middle East and Asia [1]. Dogs are the main hosts and the principal reservoir hosts of human visceral infection. Parasites are transmitted by the bites of infected phlebotomine sand fly females (Diptera: Phlebotominae) with Phlebotomus perniciosus being the main vector in southwestern Europe [2, 3].

The outcome of L. infantum infection is a consequence of intricate interactions between the protozoan and the genetic and immunological background of the host and ranges from the total absence of signs to severe systemic disease leading to death. In endemic areas, the percentage of subclinical infections is much more frequent than patent disease [4]. Despite the absence of clinical signs, subclinical dogs can serve as a source of infection for phlebotomine vectors [5]. Thus, apart from the confirmation of clinical suspicion in a single patient, the confirmation of Leishmania infection in dogs without clinical signs should be attempted to promote their monitoring through follow-up $[6,7]$.

The definitive diagnosis of CanL is complex and should integrate anamnesis, clinical, haematological and biochemical findings, as well as the detection of the parasite and/or the immune response developed by the host [8]. The commonly used laboratory techniques for the diagnosis include the direct detection of Leishmania DNA by molecular techniques such as polymerase chain reaction (PCR), and the indirect detection of antibodies against the parasite such as immunofluorescence antibody test (IFAT) or enzyme-linked immunosorbent assay (ELISA) [9]. The late appearance of specific antibodies along with the visceral tropism of the parasites makes sampling, as well as owner compliance, challenging as invasive collection of material biopsies, such as bone marrow, needs to be frequently repeated. Therefore, a diagnostic test using biological samples collected by minimal or non-invasive approaches is simpler to perform, better tolerated by animals and by far more acceptable to dog owners. The use of conjunctiva swabs as samples, coupled with a sensitive and specific PCR, has shown encouraging results for diagnosis, treatment follow-up and/or for assessing Leishmania exposure in dogs $[10,11]$. Peripheral blood sampling allows the concomitant serological and molecular diagnosis of CanL. The main disadvantage of this biological sample is the inconsistency of parasitaemia over the course of infection, especially in subclinical animals, and therefore molecular tests applied on blood samples are mostly used as a complement of serological results [9].
During the blood meal, immunogenic components present in phlebotomine sand fly saliva are inoculated into the vertebrate host provoking the development of specific anti-saliva antibodies [12]. In endemic areas of leishmaniosis caused by L. infantum, the detection of these anti-sand fly salivary antibodies has proven to be a useful epidemiological biomarker for monitoring exposure of hosts to vectors and it might be used to estimate the risk for Leishmania infection. Phlebotomus perniciosusspecific salivary recombinant proteins have been produced, to overcome difficulties in obtaining appropriate amounts of whole salivary gland homogenates (SGH), especially in large scale epidemiological studies [13]. The evaluation of different recombinant antigens of $P$. perniciosus revealed yellow-related protein (rSP03B) and/ or apyrase (rSP01) as the most promising candidates to replace SGH in the detection of $P$. perniciosus exposure in dogs [14-16] and other mammalian hosts [17].

The aim of this longitudinal study was to: (i) evaluate and compare diagnostic tests using minimally (i.e. peripheral blood) and non-invasive samples (i.e. conjunctiva cells) for monitoring Leishmania-dog infection; (ii) assess salivary antigens as markers of exposure to $P$. perniciosus and to Leishmania infection; and (iii) explore putative risks factors associated with Leishmania infection in sheltered dogs at the beginning, and the end of sand fly seasonal activity. The possible association between phlebotomine sand fly exposure and the presence of Leishmania infection was also investigated.

\section{Methods}

\section{Animals}

A total of 208 dogs (convenience sampling) from four private kennels from Lisbon and Setúbal districts belonging to the Metropolitan Lisbon region were enrolled in May and October 2011 (i.e. at the beginning and at the end of sand fly seasonal activity). CanL caused by L. infantum is endemic in both districts, and Phlebotomus ariasi and $P$. perniciosus are the proven vectors $[18,19]$. Phlebotomus sergenti and Sergentomyia minuta are the other two sand fly species known to be endemic in the study area [2]. The four kennels have an elevated turnover due to new stray dog collections, adoptions and deaths. Whenever available, data on sex, breed, coat length, coat colour, age, use of insecticides and presence of clinical signs compatible with leishmaniosis (namely muscular atrophy, cutaneous lesions, epistaxis, lameness, lymphadenomegaly, onychogryphosis, ocular lesions, pale mucous membranes or weight loss; [20]) were recorded for each dog. Blood and serum samples were obtained from previous epidemiological studies regarding exposure to $L$. infantum or Toscana and sand fly fever Sicilian viruses [21-24]. 


\section{Samples}

Peripheral blood (PB) (2-3 ml) was obtained by cephalic venipuncture from each animal and collected into serumseparating tubes and spotted on 3M filter paper. Serum was obtained by centrifugation and stored at $-20{ }^{\circ} \mathrm{C}$ until use in serological analyses and blood spotted on filter paper was preserved at $4{ }^{\circ} \mathrm{C}$ until DNA extraction.

Exfoliative epithelial cells were collected from the right and left conjunctiva (i.e. conjunctiva cells (CS)) of each animal using sterile cotton swabs. The swabs were rubbed against the surface of the lower eyelid, and then the cotton tip was immersed into a sterile $15 \mathrm{ml}$ tube containing $1 \mathrm{ml}$ of sterile saline. Twenty-four hours after incubation at $4{ }^{\circ} \mathrm{C}$, swabs were pressed against the walls of the tube, and the saline containing eluted exfoliated cells was transferred into $2 \mathrm{ml}$ sterile vials until DNA extraction [10]. Samples from each eyelid conjunctivas were processed separately.

\section{DNA extraction and PCR amplification}

A commercial kit (Kit Citogene ${ }^{\circledR}$; Citomed, Lisbons, Portugal) was used to extract DNA from blood on filter paper. Four discs of filter paper (4 $\mathrm{mm}$ in diameter each) were incubated with lysis buffer $(150 \mu \mathrm{l})$ and $1.5 \mu \mathrm{l}$ of proteinase $\mathrm{K}(20 \mathrm{mg} / \mathrm{ml})$. Further DNA extraction followed the kit manufacturer's instructions [24]. The saline samples containing eluted conjunctival cells were centrifuged at $3824 \times g$ for $10 \mathrm{~min}$, and the pellets resuspended in $90 \mu \mathrm{l}$ lysis buffer containing $10 \mu \mathrm{l}$ of $2 \mathrm{mg} / \mathrm{ml}$ proteinase $\mathrm{K}$. After $2 \mathrm{~h}$ incubation at $56{ }^{\circ} \mathrm{C}$, the samples were incubated for $10 \mathrm{~min}$ at $95{ }^{\circ} \mathrm{C}$ and then centrifuged at $17,949 \times g$ for $10 \mathrm{~min}$. DNA samples from both right and left conjunctivas were combined to increase DNA yield and stored at $-20{ }^{\circ} \mathrm{C}$ until used in the PCR assay [10].

Detection of Leishmania DNA was performed using a nested PCR (nPCR) protocol with primers targeting the small subunit ribosomal RNA (SSU-rDNA) gene [25]. A positive control containing genomic L. infantum DNA and a negative control without DNA template were included in each amplification. The DNA amplicons were resolved by conventional electrophoresis on $1.5 \%$ agarose gels stained with Green Safe Premium (Nzytech, Lisbon, Portugal), using a 100-bp DNA ladder as a molecular weight marker, then visualized under UV illumination. Positive samples yielded a predicted nPCR product of 358 bp.

\section{Detection of anti-Leishmania antibodies}

Detection of anti-Leishmania antibodies was performed by an in-house IFAT and a commercial ELISA (Bordier Affinity Products SA, Crissier, Switzerland) as previously described [26]. Briefly, a L. infantum MON-1 (MCAN/ PT/05/IMT-373) suspension of $10^{7}$ promastigotes was used as antigen, and anti-dog IgG (whole molecule)-FITC (Sigma-Aldrich, Missouri, USA) was used at a dilution of 1:20. A serum sample from a seropositive dog was used as positive control, while the serum sample of a dog from a non-endemic country of leishmaniosis (i.e. Czech Republic) and negative for Leishmania infection by both molecular and serological tests was used as negative control. The IFAT cut-off value was established at a serum dilution of 1:64. The ELISA was performed according to manufacturer's guidelines, and the result was considered positive when the absorbance of the analysed sample was higher than the absorbance of the weak positive control serum provided with the kit. The ELISA cut-off was 0.260 , according to manufacturer's instructions.

\section{Sand fly salivary proteins and detection of anti-P. perniciosus saliva antibodies}

The colony of Phlebotomus perniciosus was maintained as previously described [27]. Salivary glands, dissected from 4-6 day-old females, were pooled in $20 \mathrm{mM}$ Tris buffer with $150 \mathrm{mM} \mathrm{NaCl}$ then kept at $-80{ }^{\circ} \mathrm{C}$ until use. Recombinant salivary proteins from P. perniciosus, $35.5 \mathrm{kDa}$ apyrase (rSP01) and $43 \mathrm{kDa}$ yellow-related protein (rSP03B) were obtained from Apronex s.r.o. (Prague, Czech Republic), as previously described [13, 14].

Anti-P. perniciosus IgG was measured by ELISA as previously described [14]. Briefly, microtiter plates were coated either with salivary gland homogenate (SGH) $(0.2$ salivary gland per well) or with $\mathrm{rSP} 03 \mathrm{~B}(5 \mu \mathrm{g} / \mathrm{ml})$ or with combination of two antigens $\mathrm{rSP} 03 \mathrm{~B}+\mathrm{rSP} 01(5 \mu \mathrm{g} / \mathrm{ml}$ of each protein) in $20 \mathrm{mM}$ carbonate-bicarbonate buffer (pH 9.5) and incubated overnight at $4{ }^{\circ} \mathrm{C}$. The plates were washed with PBS $+0.05 \%$ Tween 20 (PBS-Tw) and incubated with blocking solution, $6 \%(\mathrm{w} / \mathrm{v})$ low fat dry milk diluted in PBS-Tw at $37{ }^{\circ} \mathrm{C}$ for $60 \mathrm{~min}$. Canine sera diluted 1:200 for $\mathrm{SGH}$, and 1:100 for recombinant proteins in $2 \%$ (w/v) low fat dry milk/PBS-Tw were added to the wells (100 $\mu \mathrm{l} /$ well $)$ after washing twice with PBS-Tw. After a 90 min incubation at $37{ }^{\circ} \mathrm{C}$, the plates were washed with PBS-Tw and incubated at $37{ }^{\circ} \mathrm{C}$ for $45 \mathrm{~min}$ with the secondary antibody (anti-dog IgG (whole molecule)-FITC; Sigma-Aldrich) diluted 1:9,000 in PBS-Tw. The ELISA was developed using orthophenylenediamine $(0.5 \mathrm{mg} / \mathrm{ml})$ in a phosphate citrate buffer $(\mathrm{pH} 5.5)$ with $0.001 \%$ hydrogen peroxide (30\%). Absorbance was measured at 492 nm using a NanoQuant plate reader (Infinite M200 Pro; Tecan, Männedorf, Switzerland). Each serum was tested in duplicate. Wells without serum (but coated with SGH) were used as blanks, while sera from six dogs living in a non-endemic country (Czech Republic) for leishmaniosis and without phlebotomine sand fly vectors, served as negative controls. The cut-off value was calculated by the addition of two standard deviations to the mean optical 
density (OD) of the control sera. The internal validity of ELISA assay was assured by including blanks and the same negative controls in each plate.

\section{Statistical analysis}

Descriptive statistics and an exploratory data analysis were conducted for the main variables of the dataset. The normality of the quantitative variables was assessed by both Kolmogorov-Smirnov and Shapiro-Wilk tests. Associations between qualitative variables were explored through the Chi-square test, Fisher's exact test or Freeman-Halton test. The cumulative incidence and relative risk (RR) factors for Leishmania infection (inferred by ELISA, IFAT, peripheral blood nPCR and conjunctival swab nPCR) were determined from a sample of dogs classified as not exposed (i.e. negative to all tests) at the beginning of the sand fly seasonal activity. RR values $>3$ or $<0.33$ were considered as potentially biologically important [28]. The Mann-Whitney-Wilcoxon test was used to compare the median levels of antibodies to Leishmania and P. perniciosus saliva between the beginning and end of the sand fly season. The agreement between the results of different tests performed to screen dogs for exposure to Leishmania and P. perniciosus saliva was estimated using Cohen's kappa $(k)$ test $(k<0$, disagreement; $k=0$, poor agreement; $k>0-0.20$, slight agreement; $k$ of $0.20-0.40$, fair agreement; $k$ of $>0.40-0.60$, moderate agreement; $k>0.60-0.80$, substantial agreement; $k>0.80-1.00$ excellent agreement; [28]) and McNemar's test used to test for significant differences between discordant results. Spearman's correlation coefficient $\left(r_{s}\right)$ was determined to evaluate the strength $\left(r_{s}=0\right.$, none; $r_{s}>0-0.09$, negligible; $r_{s} \geq 0.10-0.39$, weak; $r_{s} \geq 0.40-$ 0.69 , moderate; $r_{s} \geq 0.70-0.90$, strong; $r_{s}>0.90$, very strong; [29]) of relationships between serum antibodies levels against Leishmania and against $P$. perniciosus saliva. Statistical significance was determined at $\alpha \leq 0.05$. The statistical analysis was conducted using IBM $^{\circledR}$ SPSS $^{\circledR}$ Statistics v 25.0, Epitools and GraphPad Prism v 6.01.

\section{Results}

\section{Leishmania-positivity and anti-P. perniciosus antibodies} at the beginning of sand fly seasonal activity

Ninety-seven (46.6\%) of the 208 screened dogs were positive by at least one out of the four tests and were thus considered as having been exposed to Leishmania at the beginning of the sand fly seasonal activity, whereas the remaining 111 (53.4\%) were negative to all tests and classified as not exposed (Table 1). Seventy-one (34.1\%) dogs were positive by $\mathrm{PB}$ nPCR only and $4(1.9 \%)$ by ELISA only; 22 (10.6\%) dogs were positive to different combinations of techniques, but none were found to be positive by all (Table 2). Parasite DNA was detected in the peripheral
Table 1 Combined results of the serological and molecular analyses performed to assess the contact of kennelled dogs to Leishmania parasites at the beginning and end of sand fly season

\begin{tabular}{|c|c|c|c|c|c|}
\hline \multicolumn{4}{|c|}{ Diagnostic test } & \multicolumn{2}{|c|}{ Sand fly season $(n=208)$} \\
\hline ELISA & IFAT & $\begin{array}{l}\text { Peripheral } \\
\text { blood PCR }\end{array}$ & $\begin{array}{l}\text { Conjunctival } \\
\text { swab PCR }\end{array}$ & Start, $n(\%)$ & End, $n(\%)$ \\
\hline- & - & - & - & $111(53.4)$ & $92(44.2)$ \\
\hline- & - & - & + & $0(0)$ & 47 (22.6) \\
\hline- & - & + & - & $71(34.1)$ & $24(11.5)$ \\
\hline- & + & - & - & $0(0)$ & $0(0)$ \\
\hline+ & - & - & - & $4(1.9)$ & $5(2.4)$ \\
\hline- & - & + & + & $0(0)$ & $15(7.2)$ \\
\hline- & + & - & + & $0(0)$ & $1(0.5)$ \\
\hline- & + & + & - & $0(0)$ & $0(0)$ \\
\hline+ & - & - & + & $0(0)$ & $4(1.9)$ \\
\hline+ & - & + & - & $8(3.8)$ & $0(0)$ \\
\hline+ & + & - & - & $8(3.8)$ & $9(4.3)$ \\
\hline- & + & + & + & $0(0)$ & $0(0)$ \\
\hline+ & + & + & - & 62.9) & $6(2.9)$ \\
\hline+ & + & - & + & $0(0)$ & $4(1.9)$ \\
\hline+ & - & + & + & $0(0)$ & $1(0.5)$ \\
\hline+ & + & + & + & $0(0)$ & $0(0)$ \\
\hline
\end{tabular}

a All negative at the beginning of the sand fly season

Abbreviations: ELISA, enzyme-linked immunosorbent assay; IFAT, immunofluorescence antibody test; $P C R$, polymerase chain reaction

blood of 85 (40.9\%) dogs but in none of the conjunctival samples. Antibodies to Leishmania were detected in $26(12.5 \%)$ sera by ELISA and in $14(6.7 \%)$ sera by IFAT. ELISA OD values ranged between $0-1.680$, while IFAT titres ranged between 0-2048 (Table 3). According to ELISA results, the presence of antibodies to the parasite was significantly higher in pure breed dogs in comparison to mongrels $(P=0.019)$, and in those dogs presenting clinical signs compatible with leishmaniosis $(P=0.035)$ (Table 2). No significant differences were detected in positivity to Leishmania by IFAT or PB nPCR among all the variables studied.

One hundred seventy-nine (86.1\%) dogs were seropositive to $P$. perniciosus saliva (Table 4). Antibodies to rSP03B + rSP01, SGH and rSP03B were detected in 174 (83.7\%), 155 (74.5\%) and 129 (62.0\%) sera, respectively. One hundred twenty-one (58.2\%) dogs were positive to the three salivary antigens, while 58 (27.9\%) dogs were positive to different combinations of them. The OD values and frequency distribution for each $P$. perniciosus salivary antigen are summarized in Table 3.

A substantial agreement was found between ELISA and IFAT for the detection of antibodies against the parasite $(k=0.671)$ and a moderate agreement was obtained between the three salivary antigens $(k=0.484$ for rSP03B 


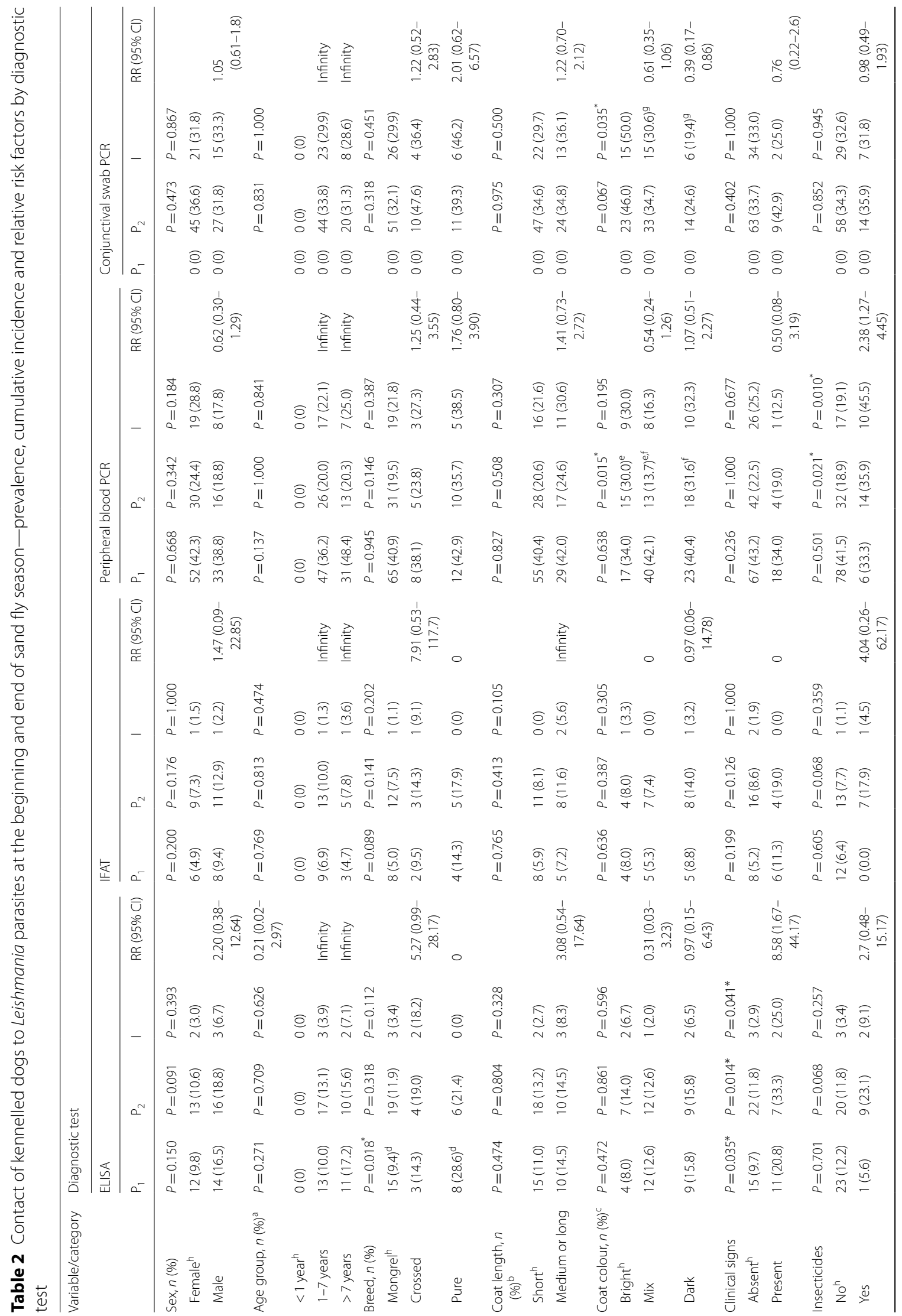




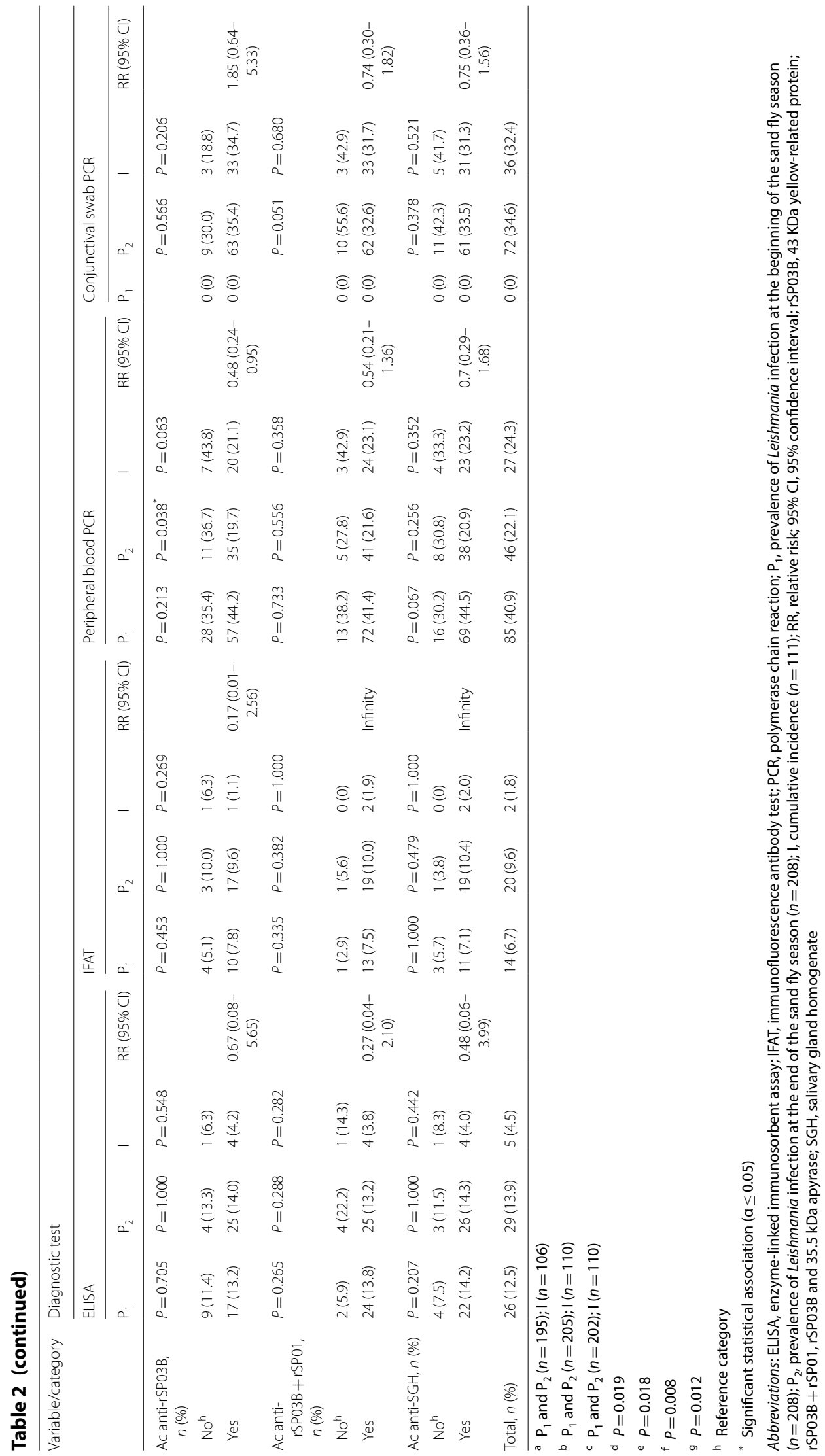


Table 3 Comparison of median levels of antibodies to Leishmania or Phlebotomus perniciosus at the beginning and end of the sand fly season

\begin{tabular}{|c|c|c|c|c|c|}
\hline \multirow[t]{3}{*}{ Serological technique } & \multicolumn{4}{|c|}{ Sand fly season $(n=208)$} & \multirow[t]{3}{*}{$P$-value } \\
\hline & \multicolumn{2}{|l|}{ Start } & \multicolumn{2}{|l|}{ End } & \\
\hline & Median (IQR) & Range & Median (IQR) & Range & \\
\hline \multicolumn{6}{|l|}{ P. perniciosus } \\
\hline ELISA-rSP03B & $0.350(0.282-0.496)$ & $0.117-1.406$ & $0.369(0.280-0.491)$ & $0.143-1.066$ & 0.793 \\
\hline ELISA-rSP03B + rSP01 & $0.370(0.292-0.508)$ & $0.129-1.253$ & $0.393(0.308-0.556)$ & $0.164-1.451$ & 0.060 \\
\hline ELISA-SGH & $0.237(0.186-0.343)$ & $0.083-1.444$ & $0.282(0.232-0.380)$ & $0.110-1.404$ & $<0.001$ \\
\hline \multicolumn{6}{|l|}{ Leishmania } \\
\hline ELISA & $0.037(0.024-0.064)$ & $0.000-1.680$ & $0.038(0.025-0.071)$ & $0-1.620$ & 0.457 \\
\hline IFAT & $0(0-0)$ & 0-2048 & $0(0-0)$ & 0-9192 & 0.919 \\
\hline
\end{tabular}

Abbreviations: ELISA, enzyme-linked immunosorbent assay; IFAT, immunofluorescence antibody test; rSP03B, 43 KDa yellow-related protein; rSP03B + rSP01, rSP03B and $35.5 \mathrm{kDa}$ apyrase; SGH, salivary gland homogenate; IQR, interquartile interval (quartile 1-quartile 3)

Table 4 Combined results of the serological analyses performed to assess the exposure of kennelled dogs to Phlebotomus perniciosus at the beginning and end of the sand fly season

\begin{tabular}{|c|c|c|c|c|}
\hline \multicolumn{3}{|c|}{ Salivary gland antigen } & \multicolumn{2}{|c|}{ Sand fly season $(n=208)$} \\
\hline rSP03B & rSP03B + rSP01 & SGH & Start, $n(\%)$ & End, $n(\%)$ \\
\hline- & - & - & $29(13.9)$ & $10(4.8)$ \\
\hline- & - & + & $5(2.4)$ & $5(2.4)$ \\
\hline- & + & - & $16(7.7)$ & $6(2.9)$ \\
\hline+ & - & - & $0(0)$ & $2(1.0)$ \\
\hline- & + & + & $29(13.9)$ & $9(4.3)$ \\
\hline+ & - & + & $0(0)$ & $1(0.5)$ \\
\hline+ & + & - & $8(3.8)$ & $8(3.8)$ \\
\hline+ & + & + & $121(58.2)$ & $167(80.3)$ \\
\hline
\end{tabular}

Abbreviations: rSP03B, 43 KDa yellow-related protein; rSP03B + rSP01, rSP03B and $35.5 \mathrm{kDa}$ apyrase; SGH, salivary gland homogenate

and $\mathrm{rSP} 03 \mathrm{~B}+\mathrm{rSP} 01 ; k=0.542$ for $\mathrm{rSP} 03 \mathrm{~B}$ and $\mathrm{SGH}$; $k=0.584$ for rSP03B + rSP01 and SGH) (Table 5). Discordant results were found between the different serological techniques for the detection of Leishmania- and $P$. perniciosus-specific antibodies and $\mathrm{PB} \mathrm{nPCR}$.

Significant positive correlations between the antibody levels to the three salivary antigens were observed $\left(r_{s}=0.928, \quad P<0.001\right.$, between $\mathrm{rSP} 03 \mathrm{~B}+\mathrm{rSP} 01$ and rSP03B; $r_{s}=0.841, P<0.001$, between $\mathrm{rSP} 03 \mathrm{~B}+\mathrm{rSP} 01$ and SGH; $r_{s}=0.820, P<0.001$, between rSP03B and $\mathrm{SGH}$ ) (Additional file 1: Figure S1). The OD of the antibody levels to the three salivary antigens and to both $\mathrm{rSP} 03 \mathrm{~B}+\mathrm{rSP} 01 / \mathrm{SGH}$ were found to be positively correlated with the ELISA OD (rSP03B: $r_{s}=0.244, P<0.001$; rSP03B + rSP01: $r_{s}=0.286, P<0.001 ; \quad \mathrm{SGH}: r_{s}=0.250$, $P<0.001)$ and IFAT titres (rSP03B + rSP01: $r_{s}=0.146$, $P<0.035$; SGH: $\left.r_{s}=0.178, P<0.010\right)$ to $L$. infantum, respectively. The increase in ELISA OD levels of antibodies to Leishmania was also significantly correlated with the increase in IFAT titres $\left(r_{s}=0.473, P<0.001\right)$.

\section{Leishmania-positivity and anti-P. perniciosus antibodies at the end of sand fly seasonal activity}

At the end of sand fly seasonal activity 116 (55.8\%) of the 208 screened dogs were positive by at least one test, while $92(44.2 \%)$ remained negative (Table 1). Fortyseven (22.6\%) dogs were positive by CS nPCR only, 24 (11.5\%) by PB nPCR only, and 5 (2.4\%) by ELISA only; $39(18.8 \%)$ dogs were positive to different combinations of techniques, but none were found to be positive by all. Parasite DNA was detected in the peripheral blood and conjunctival samples of $46(22.1 \%)$ and $72(34.6 \%)$ dogs, respectively. The PB nPCR result was found significantly associated with coat colour $(P=0.015)$, insecticide treatment $(P=0.021)$ and antibodies to rSP03B salivary antigen $(P=0.038)$ (Table 3$)$. The presence of Leishmania DNA was significantly higher in dogs with light $(P=0.018)$ or dark $(P=0.008)$ coat in comparison to those with mix colour coat, in dogs treated with insecticides $(P=0.021)$ and in those lacking specific antibodies to $\mathrm{rSP03B}(P=0.038)$. Dogs without significant levels of antibodies to this salivary antigen had a RR 0.48 times lower (95\% CI: $0.24-0.95$ ) to be found positive by PB $\mathrm{nPCR}$. The incidence of the detection of parasite DNA in the peripheral blood and conjunctival cells was significantly higher in dogs treated with insecticides $(P=0.010$, $\mathrm{RR}=2.38,95 \% \mathrm{CI}: 1.25-4.45)$ and with coloured coat $(P=0.035)$, respectively. Further, dogs with dark coat had a RR 0.39 times lower (95\% CI: $0.17-0.86$ ) to be found positive by CS nPCR than dogs with light coat.

Antibodies to Leishmania were detected in 29 (13.9\%) sera by ELISA and in $20(9.6 \%)$ sera by IFAT. The ELISA 
Table 5 Agreement rates between diagnostic tests results at the beginning and end of the sand fly season

\begin{tabular}{|c|c|c|c|c|c|c|c|c|c|c|c|c|}
\hline \multirow[t]{3}{*}{ Comparison } & \multicolumn{12}{|c|}{ Sand fly season } \\
\hline & \multicolumn{6}{|l|}{ Start } & \multicolumn{6}{|l|}{ End } \\
\hline & $P$-value & Kappa & $95 \% \mathrm{Cl}$ & PA (\%) & NA (\%) & $\mathrm{OA}(\%)$ & $P$-value & Kappa & $95 \% \mathrm{Cl}$ & PA (\%) & NA (\%) & $\mathrm{OA}(\%)$ \\
\hline$A v s B$ & 0.002 & 0.671 & $0.501-0.842$ & 70.0 & 98.8 & 94.2 & 0.016 & 0.747 & $0.605-0.888$ & 77.6 & 97.0 & 94.7 \\
\hline Avs C & $<0.001$ & 0.075 & $-0.031-0.181$ & 25.2 & 72.8 & 60.1 & 0.041 & 0.019 & $-0.115-0.153$ & 18.7 & 82.1 & 70.7 \\
\hline$A v s D^{a}$ & & & & & & & $<0.001$ & -0.026 & $-0.138-0.087$ & 17.8 & 73.7 & 60.1 \\
\hline Avs $E$ & $<0.001$ & 0.014 & $-0.059-0.009$ & 21.9 & 53.6 & 41.2 & $<0.001$ & 0.002 & $-0.041-0.045$ & 24.2 & 24.9 & 24.5 \\
\hline$A v s F$ & $<0.001$ & 0.029 & $-0.007-0.064$ & 24.0 & 29.6 & 26.9 & $<0.001$ & -0.018 & $-0.058-0.022$ & 22.8 & 14.2 & 18.8 \\
\hline$A \vee s G$ & $<0.001$ & 0.037 & $-0.014-0.088$ & 24.3 & 41.7 & 34.1 & $<0.001$ & 0.008 & $-0.030-0.046$ & 35.3 & 24.6 & 23.6 \\
\hline B vs C & $<0.001$ & 0.006 & $-0.074-0.089$ & 12.2 & 72.6 & 58.2 & $<0.001$ & 0.055 & $-0.078-0.188$ & 18.2 & 84.6 & 74.0 \\
\hline $\mathrm{B} v s \mathrm{D}^{\mathrm{a}}$ & & & & & & & $<0.001$ & -0.049 & $-0.144-0.046$ & 10.9 & 74.7 & 60.6 \\
\hline$B v s E$ & $<0.001$ & 0.021 & $-0.031-0.073$ & 14.0 & 55.0 & 40.9 & $<0.001$ & -0.001 & $-0.038-0.035$ & 17.2 & 24.8 & 21.2 \\
\hline$B$ vs $F$ & $<0.001$ & 0.016 & $-0.009-0.040$ & 13.8 & 29.0 & 22.1 & $<0.001$ & 0.008 & $-0.014-0.030$ & 18.1 & 16.5 & 17.3 \\
\hline$B v s G$ & $<0.001$ & 0.008 & $-0.032-0.047$ & 13.0 & 40.5 & 29.3 & $<0.001$ & 0.018 & $-0.007-0.043$ & 18.8 & 23.4 & 21.2 \\
\hline$C v s D^{a}$ & & & & & & & 0.007 & 0.002 & $-0.132-0.132$ & 27.1 & 71.1 & 58.7 \\
\hline CVsE & $<0.001$ & 0.079 & $-0.044-0.202$ & 53.3 & 50.5 & 51.9 & $<0.001$ & -0.060 & $-0.128-0.008$ & 31.3 & 19.8 & 26.0 \\
\hline CVsF & $<0.001$ & 0.015 & $-0.072-0.103$ & 55.6 & 26.8 & 44.7 & $<0.001$ & -0.013 & $-0.060-0.034$ & 34.8 & 14.4 & 26.0 \\
\hline Cvs G & $<0.001$ & 0.010 & $-0.004-0.204$ & 57.5 & 42.1 & 51.0 & $<0.001$ & -0.031 & $-0.089-0.028$ & 33.3 & 19.2 & 26.9 \\
\hline$D v s E^{a}$ & & & & & & & $<0.001$ & 0.022 & $-0.051-0.095$ & 50.4 & 25.3 & 40.4 \\
\hline$D v s F^{a}$ & & & & & & & $<0.001$ & -0.058 & $-0.123-0.008$ & 47.3 & 10.4 & 33.7 \\
\hline$D v s G^{a}$ & & & & & & & $<0.001$ & -0.031 & $-0.104-0.041$ & 48.0 & 18.5 & 36.5 \\
\hline Evs F & $<0.001$ & 0.484 & $0.369-0.598$ & 85.2 & 60.2 & 78.4 & 0.010 & 0.580 & $0.408-0.752$ & 95.1 & 62.5 & 91.4 \\
\hline Evs $G$ & $<0.001$ & 0.542 & $0.425-0.660$ & 85.2 & 68.2 & 79.8 & 0.540 & 0.505 & $0.334-0.677$ & 93.3 & 54.1 & 88.5 \\
\hline$F \vee s G$ & $<0.001$ & 0.584 & $0.451-0.716$ & 91.2 & 66.7 & 86.1 & 0.118 & 0.494 & $0.304-0.684$ & 94.6 & 54.6 & 90.4 \\
\hline
\end{tabular}

a Leishmania DNA was not detected at the beginning of the phlebotomine sand fly season by conjunctival swab PCR

Abbreviations: A, enzyme-linked immunosorbent assay; $B$, immunofluorescence antibody test; $C$, peripheral blood-polymerase chain reaction (PCR); $D$, conjunctival swab-PCR; E, $43 \mathrm{KDa}$ yellow-related protein; F, 43 kDa yellow-related protein and $35.5 \mathrm{kDa}$ apyrase; G, salivary gland homogenate; PA, positive agreement; NA, negative agreement; $\mathrm{OA}$, overall agreement

result was significantly associated with clinical signs $(P=0.014)$. Dogs presenting clinical signs compatible with leishmaniosis had a RR 8.58 times higher $(95 \% \mathrm{CI}$ : 1.67-44.17) to be seropositive by ELISA than those without clinical manifestations. Seroreversion in ELISA and IFAT occurred in $15.4 \%(4 / 26)$ and $7.1 \%(1 / 14)$ dog sera, respectively. Further, seroconversion was approximately $3 \%$ in both techniques: ELISA: $3.8 \%$ (7/182); IFAT: $3.1 \%$ $(6 / 194)$.

One hundred ninety-eight (95.2\%) dogs were seropositive to $P$. perniciosus saliva (Table 4). Antibodies to rSP03B + rSP01, SGH and rSP03B were detected in 190 (91.3\%), $182(87.5 \%)$ and 178 (85.6\%), respectively. One hundred sixty-seven (80.3\%) dogs were positive to the three salivary antigens, while 31 dogs were positive to different combinations of them. The OD values and frequency distribution for each $P$. perniciosus salivary antigen are summarized in Table 2.

A substantial agreement was found between ELISA and IFAT for the detection of antibodies against the parasite $(k=0.747)$ and a moderate agreement was obtained between the three salivary antigens $(k=0.580$ for $\mathrm{rSP} 03 \mathrm{~B}$ and $\mathrm{rSP} 03 \mathrm{~B}+\mathrm{rSP} 01 ; k=0.505$ for $\mathrm{rSP} 03 \mathrm{~B}$ and $\mathrm{SGH}$; $k=0.494$ for $\mathrm{rSP} 03 \mathrm{~B}+\mathrm{rSP} 01$ and SGH) (Table 5). Discordant results were found between the different serological techniques for the detection of Leishmania- and $P$. perniciosus-specific antibodies, and for the molecular detection of parasite DNA.

Significant positive correlations between the antibody levels to the three salivary antigens were observed (between rSP03B +rSP01 and rSP03B: $r_{s}=0.932$, $P<0.001$; between rSP03B + rSP01 and SGH: $r_{s}=0.730$, $P<0.001$; between rSP03B and SGH: $\left.r_{s}=0.719, P<0.001\right)$ (Additional file 2: Figure S2). A significant positive correlation between $\mathrm{OD}$ values to the three salivary antigens and ELISA OD (rSP03B + rSP01: $r_{s}=0.140, P<0.140$; rSP03B: $r_{s}=0.153, P<0.028 ;$ SGH: $\left.r_{s}=0.232, P=0.001\right)$ to L. infantum was also found. The increase in ELISA OD levels of antibodies to Leishmania was significantly correlated with the increase in IFAT titre $\left(r_{s}=0.584, P<0.001\right)$. 


\section{Discussion}

In endemic areas of zoonotic leishmaniosis caused by $L$. infantum, both clinical and subclinical dogs represent a source of parasites to the vectors, contributing to the maintenance of the endemicity of the disease [5]. Therefore, early detection of Leishmania infection in both groups of infected dogs (i.e. with and without clinical signs) is essential to control the dissemination of the parasite to other dogs and humans [7, 30].

Despite clinical staging of CanL is still a matter of debate $[8,31,32]$, the diagnosis of disease in clinical dogs from endemic areas is easier to achieve than the assessment of L. infantum infection in subclinical dogs [33, 34]. The confirmation of cryptic infections is difficult due to the absence of gold standards for their diagnosis, and due to the lack of compliance of owners to screen the presence of the parasite in their dogs, considered by them as "healthy", especially when sampling is invasive and needs to be frequently repeated $[9,10,30]$.

The most useful diagnostic approaches for the investigation of infection include the detection of specific serum anti-leishmanial antibodies and parasite DNA in tissues by molecular techniques [9]. Thus, in the present study, we evaluated the molecular and serological performance of blood, a minimally invasive biological sample, to detect the presence of Leishmania antibodies or its DNA in sheltered dogs at the beginning and end of the sand fly activity period. Antibodies to Leishmania were detected in $26(12.5 \%)$ and 29 (13.9\%) sera by ELISA, and in $14(6.7 \%)$ and in $20(9.6 \%)$ sera by IFAT, at the beginning and the end of the sand fly season, respectively, reinforcing the endemicity of the parasitosis in Metropolitan region of Lisbon $[15,23]$. The increased number of seropositive dogs detected by both techniques at the end of the study might reflect on one hand, an infection that was not detectable at the start of the study, due to the low production of antibodies at the beginning of Leishmania infection [35]; this hypothesis is highly probable as most of the sheltered dogs were previously stray living in deficient health and nutritional conditions, and representing, therefore, an easier target for phlebotomine sand fly biting and for infection. On the other hand, the increased number of seropositive dogs at the end of sand fly season might represent an infection that occurred in the kennel, as the seroconversion rate by ELISA and IFAT was $4.5 \%$ and $1.8 \%$, respectively, based on incidence values. Both techniques have been shown to have a high sensitivity as they were able to detect canine antibodies to the parasite between one to five months after experimental infection of dogs with L. infantum [26]. Further, L. infantum vectors were collected in the same year of the study in the screened area [2], and thus dogs could have been bitten by infected sand flies. Despite the overall increase in the number of dogs considered seropositive at the end of the sand fly season, some animals that were positive at the beginning of the study seroreverted. Seroreversion based on different diagnostic techniques over time has already been reported among naturally infected dogs [36] which may reflect exposure to the parasite followed by its clearance [37].

Both serological techniques presented a substantial agreement and positive correlation at both sampling points supporting that they can equally be used in the serological diagnosis of this parasitosis; also, and as both are quantitative, they can be used to follow-up antibody production and response to treatment $[9,31]$. The presence of antibodies to Leishmania detected by ELISA was significantly higher in pure breed dogs in comparison to mongrels, corroborating that the latter seem to have developed a certain resistance to Leishmania infection [38]. More importantly, an association between ELISA positivity and clinical signs was observed, with dogs presenting clinical signs compatible with leishmaniosis having a RR 8.58 times higher to be seropositive by this technique than those without clinical manifestations, supporting its use to confirm a clinical suspicion. In fact, in a previous study on experimentally infected dogs, the association between clinical signs and seropositivity to this commercial ELISA was suggested as a predictive marker of the development of infection to disease [26].

Dogs not presenting antibodies to $P$. perniciosus rSP03B protein had a RR 0.48 times lower to be found positive by PB nPCR than those having this anti-sand fly saliva antigen, suggesting that the lack of canine antibodies to this specific salivary protein reflects the lower probability to encounter infected bites.

The incidence of infection measured by PB nPCR was $24.3 \%$, and it was significantly higher in dogs treated with insecticides. Despite the use of topical insecticides, which has been shown to be effective in reducing the incidence of Leishmania infection in dogs [39], the significantly higher probability of dogs treated with ectoparasiticides to harbour parasite DNA in the peripheral blood is not entirely surprising, as neither the compliance of application, nor the correct administration and effectivity of insecticides were evaluated. Whether the obtained incidence was due to transient or to real infection could not be verified, as the 27 dogs that became positive by PB nPCR at the end of the sand fly season were not followed-up further. Nevertheless, and due to the discrepancy of positive PB nPCR results at the beginning and end of sand fly seasonal activity (Table 2), together with the insufficient data regarding the duration and consistency of parasitaemia over the course of infection $[10,11$, 26, 40], DNA-positivity in PB should not be used as sole diagnostic tools for disease diagnosis. 
The diagnostic performance of conjunctiva cells obtained non-invasively, coupled with nPCR to detect the presence of Leishmania DNA was also evaluated, yielding positive results only at the end of phlebotomine seasonal activity. Out of the 72 dogs harbouring parasite DNA, 47 were positive by CS nPCR only; this situation has been reported previously in naturally and experimentally infected dogs and related to a recent parasite contact $[11,40,41]$. Whether the contact with the parasite would evolve to the clearance or establishment of infection could not be analysed as dogs were not followed-up after October. Nevertheless, CS nPCR seems to be effective for assessing early exposure to the parasite. An association between the incidence of the detection of parasite DNA in conjunctival cells and colour of coat was observed, with dogs presenting dark coat having a RR 0.39 times lower by this technique than those with light coat. The attractiveness of phlebotomine sand flies to different light colours of CDCs traps has been demonstrated [42]; however, it is currently unknown whether the eyes of $L$. infantum vectors react more to light coat than to dark.

Overall, the discordance found between the serological and molecular techniques for determining Leishmania infection/exposure and incidence confirms that they do not have the same diagnostic performance and reinforces that Leishmania-dog contact should be monitored using more than one technique $[9,11,34]$. The early detection of parasite contact will allow dogs to be followed-up to confirm the clearance or establishment of infection, and to adopt control measures to avoid parasite transmission to the vectors.

The quantification of anti-P. perniciosus saliva antibodies in vertebrate hosts of $L$. infantum has proven to be an effective way of measuring exposure to this parasite vector $[14-17,43,44]$. As in the year that the study was conducted, the sand fly activity in the Metropolitan region of Lisbon started in May and ended in October [2], it is not surprisingly the high overall levels of seropositivity to anti-sand fly saliva antigens at the beginning (86.1\%) and at the end (95.2\%) of sand fly season, confirming the CanL endemicity status for the region [24]. As antibodies to sand fly saliva decay after the end of the biting season [14], the detection of antibodies to P. perniciosus saliva in a high percentage of dogs at the beginning of the season was probably related to their re-exposure to sand flies following antigenic priming in the previous season [15]. On the other hand, and since antibodies against saliva rise during summer months when sand fly abundance is higher [14], the increased number of dogs seropositive to salivary antigens at the end of the sand fly season was probably related with the exposure to more sand fly bites, as a second peak of P. perniciosus activity in Portugal was observed in September [2].
Antibodies recognizing SGH, rSP03B and rSP03B + rSP01 followed similar dynamics throughout the study reinforcing their use to assess exposure to $P$. perniciosus in dogs living in L. infantum endemic areas. A combination of the two recombinant proteins (rSP03B + rSP01) showed a better performance and higher mean OD values than the single rSP03B to detected dogs exposure to P. perniciosus. Similar results were previously published [13], while other studies on $P$. perniciosus prefer to use a single antigen rSP03B [14-16]. In Lutzomyia longipalpis, the main vector of $L$. infantum in the New World, a combination of two recombinant salivary proteins were successfully used [45].

In the study area, $P$. perniciosus is sympatric with $P$. ariasi [2], a closely related species of the subgenus Larroussius; thus, the high percentage of detection of antibodies to SGH might reflect cross-reactivity with antibodies against $P$. ariasi $[12,16]$. As the presence and abundance of sand fly species responsible for $L$. infantum transmission varies according to the location, and throughout the transmission season, the sole use of SGH to specifically measure exposure to $P$. perniciosus, the most abundant L. infantum vector in the Western Mediterranean, can be hampered; however, this cross-reactivity might be an advantage, as both Larroussius species are proven $L$. infantum vectors [3].

The use of antibodies to sand fly salivary antigens as risk markers of L. infantum infection in dogs has been repeatedly evaluated but remains controversial: positive [14-16], negative [43] or no correlations [15] between the levels of anti-P. perniciosus saliva and L. infantum infection have been reported in dogs from endemic areas of leishmaniosis. As the antigenic response to phlebotomine sand fly saliva reflects sand fly bites, whether the sand fly is infected or not, while Leishmania infection only occurs if the vertebrate host after being inoculated with the parasite cannot clear it, the relationship between the dynamics of antibodies against sand fly saliva and a subsequent Leishmania infection can only be evaluated in longitudinal studies. In the present study, a significant positive (although low) correlation between OD values to the three salivary antigens and ELISA OD to L. infantum was found in both time intervals and between IFAT titres and OD values to salivary antigens at the beginning of the phlebotomine season, reinforcing their potential usefulness as biomarkers of $L$. infantum infection $[15,16,46]$.

\section{Conclusions}

The association between ELISA positivity and clinical signs compatible with leishmaniosis suggests its use to confirm a clinical suspicion. CS nPCR seems to be an effective and non-invasive method for assessing early 
exposure to the parasite. Salivary antigens are useful to monitor dog exposure to P. perniciosus bites in areas where this sand fly species is present. The correlation between the levels of antibodies to P. perniciosus saliva and Leishmania antibodies suggests the use of canine humoral response to salivary antigens as biomarkers of $L$. infantum infection.

\section{Supplementary information}

Supplementary information accompanies this paper at https://doi. org/10.1186/s13071-020-3993-7.

Additional file 1: Figure S1. Correlations between serum antibodies levels at the beginning of sand fly season. a Between $43 \mathrm{KDa}$ yellowrelated protein ( $r S P 03 B)$ and $r S P 03 B+35.5 \mathrm{kDa}$ apyrase ( $\mathrm{SP} 03 \mathrm{~B}+\mathrm{rSP} 01)$. b Between $\mathrm{rSP} 03 \mathrm{~B}$ and salivary gland homogenate $(\mathrm{SGH})$. c Between rSP03B and Leishmania [enzyme-linked immunosorbent assay (ELISA)]. d Between rSP03B and Leishmania immunofluorescence antibody test (IFAT). e Between rSP03B + rSP01 and SGH. f Between rSP03B + rSP01 and Leishmania-ELISA. g Between rSPO3B + rSP01 and Leishmania-IFAT. $\mathbf{h}$ Between SGH and Leishmania-ELISA. i Between SGH and Leishmania-IFAT. j Between Leishmania-ELISA and Leishmania-IFAT. Abbreviation: OD, optical density.

Additional file 2: Figure S2. Correlations between serum antibodies levels at the end of sand fly season. a Between $43 \mathrm{KDa}$ yellow-related protein (rSP03B) and rSP03B + $35.5 \mathrm{kDa}$ apyrase (rSP03B + rSP01). b Between $\mathrm{rSP} 03 \mathrm{~B}$ and salivary gland homogenate (SGH). c Between rSP03B and Leishmania [enzyme-linked immunosorbent assay (ELISA)] d Between $\mathrm{rSP} 03 \mathrm{~B}$ and Leishmania immunofluorescence antibody test (IFAT). e Between rSP03B + rSP01and SGH. $\mathbf{f}$ between rSP03B + rSP01 and Leishmania-ELISA. g Between rSPO3B + rSP01 and Leishmania-IFAT. $\mathbf{h}$ Between SGH and Leishmania-ELISA. i Between SGH and Leishmania-IFAT. j Between Leishmania-ELISA and Leishmania-IFAT. Abbreviation: OD, optical density.

\section{Abbreviations}

CanL: canine leishmaniosis; Cl: confidence interval; CS: conjunctival cells; ELISA: enzyme-linked immunosorbent assay; IFAT: immunofluorescence antibody test; nPCR: nested PCR; OD: optical density; PB: peripheral blood; $P C R$ : polymerase chain reaction; $R R$ : relative risk; rSP01: recombinant apyrase; rSP03B: recombinant yellow-related protein; SGH: salivary gland homogenate.

\section{Acknowledgements}

The authors would like to thank legal holders, staff members and volunteers of the shelters for their assistance. Publication of this paper has been sponsored by Bayer Animal Health in the framework of the 15th CVBD ${ }^{\circledR}$ World Forum Symposium.

\section{Authors' contributions}

CM supervised the study, collected conjunctival exfoliative cells and information of each dog, performed ELISAs and wrote the manuscript. JMC collected blood samples and performed IFAT, ELISAs and molecular analysis. AP performed statistical analysis and helped to write the draft; TK, TS and PS dissected P. perniciosus salivary glands and prepared SGH, rSP01 and rSP03B; PV and LC critically reviewed the manuscript. All authors read and approved the final manuscript.

\section{Funding}

Fundação para a Ciência e a Tecnologia, I.P. (FCT) through contract GHTM-UID/ Multi/04413/2013. AP and CM have the support of the Portuguese Ministry of Education and Science (via Fundação para a Ciência e a Tecnologia, I.P.), through a PhD grant (SFRH/BD/116516/2016) and an Investigator Starting Grant IF/01302/2015, respectively. PV, TS and TL are supported by ERD funds, project CeRaViP (16_019/0000759) and UNCE 204072.

\section{Availability of data and materials}

The data supporting the conclusions of this article are included within the article and its Additional files.

\section{Ethics approval and consent to participate}

The procedures were approved by the Ethical Committee of IHMT (authorization no. 8 2011-PI) as complying with the Portuguese legislation for the protection of animals (Decree-Law No. 113/2013). Consent was obtained from the legal detainer, i.e. the person in charge of the private shelters.

\section{Consent for publication}

Not applicable.

\section{Competing interests}

The authors declare that they have no competing interests.

\section{Author details}

${ }^{1}$ Global Health and Tropical Medicine (GHTM), Instituto de Higiene e Medicina Tropical (IHMT), Universidade NOVA de Lisboa (UNL), Lisbon, Portugal. ${ }^{2}$ Medical Parasitology Unit, IHMT-UNL, Lisbon, Portugal. ${ }^{3}$ Department of Parasitology, Faculty of Science, Charles University in Prague, Prague, Czech Republic.

Received: 4 November 2019 Accepted: 24 February 2020

Published online: 21 April 2020

\section{References}

1. WHO. Control of the leishmaniases: report of a meeting of the $\mathrm{WHO}$ expert commitee on the control of leishmaniases, Geneva, 22-26 March 2010. Geneva: World Health Organization; 2010.

2. Alten B, Maia C, Afonso MO, Campino L, Jiménez M, González E, et al. Seasonal dynamics of phlebotomine sand fly species proven vectors of mediterranean leishmaniasis caused by Leishmania infantum. PLoS Negl Trop Dis. 2016;10:e0004458.

3. Dvorak V, Shaw J, Volf P. Parasite biology: the vectors. In: Bruschi F, Gradoni L, editors. The Lleishmaniases: old neglected and tropical diseases. Austria: Springer; 2018. p. 31-77.

4. Baneth G, Koutinas AF, Solano-Gallego L, Bourdeau P, Ferrer L. Canine leishmaniosis - new concepts and insights on an expanding zoonosis: part one. Trends Parasitol. 2008;24:324-30.

5. Quinnell RJ, Courtenay O. Transmission, reservoir hosts and control of zoonotic visceral leishmaniasis. Parasitology. 2009;136:1915-34.

6. Solano-Gallego L, Cardoso L, Pennisi MG, Petersen C, Bourdeau P, Oliva $G$, et al. Diagnostic challenges in the era of canine Leishmania infantum vaccines. Trends Parasitol. 2017;33:706-17.

7. Maia C, Campino L. Biomarkers associated with Leishmania infantum exposure, infection, and disease in dogs. Front Cell Infect Microbiol. 2018;8:302.

8. Paltrinieri S, Gradoni L, Roura X, Zatelli A, Zini E. Laboratory tests for diagnosing and monitoring canine leishmaniasis. Vet Clin Pathol. 2016:45:552-78.

9. Maia C, Campino L. Methods for diagnosis of canine leishmaniasis and immune response to infection. Vet Parasitol. 2008;158:274-87.

10. Gramiccia M, Di Muccio T, Fiorentino E, Scalone A, Bongiorno G, Cappiello $\mathrm{S}$, et al. Longitudinal study on the detection of canine Leishmania infections by conjunctival swab analysis and correlation with entomological parameters. Vet Parasitol. 2010;171:223-8.

11. Di Muccio T, Veronesi F, Antognoni MT, Onofri A, Piergili Fioretti D, Gramiccia M. Diagnostic value of conjunctival swab sampling associated with nested PCR for different categories of dogs naturally exposed to Leishmania infantum infection. J Clin Microbiol. 2012;50:2651-9.

12. Lestinova T, Rohousova I, Sima M, de Oliveira CI, Volf P. Insights into the sand fly saliva: blood-feeding and immune interactions between sand flies, hosts, and Leishmania. PLoS Negl Trop Dis. 2017;11:e0005600.

13. Drahota J, Martin-Martin I, Sumova P, Rohousova I, Jimenez M, Molina R, et al. Recombinant antigens from Phlebotomus perniciosus saliva as markers of canine exposure to visceral leishmaniases vector. PLoS Negl Trop Dis. 2014;8:e2597. 
14. Kostalova T, Lestinova T, Sumova P, VIkova M, Rohousova I, Berriatua E, et al. Canine antibodies against salivary recombinant proteins of $\mathrm{Phle-}$ botomus perniciosus: a longitudinal study in an endemic focus of canine leishmaniasis. PLoS Negl Trop Dis. 2015;9:e0003855.

15. Kostalova T, Lestinova T, Maia C, Sumova P, Vlkova M, Willen L, et al. The recombinant protein $\mathrm{rSP03B}$ is a valid antigen for screening dog exposure to Phlebotomus perniciosus across foci of canine leishmaniasis. Med Vet Entomol. 2017;31:88-93.

16. Velez R, Spitzova T, Domenech E, Willen L, Cairó J, Volf $P$, et al. Seasonal dynamics of canine antibody response to Phlebotomus perniciosus saliva in an endemic area of Leishmania infantum. Parasites Vectors. 2018;11:545,

17. Martín-Martín I, Molina R, Rohoušová I, Drahota J, Volf P, Jiménez M. High levels of anti-Phlebotomus perniciosus saliva antibodies in different vertebrate hosts from the re-emerging leishmaniosis focus in Madrid, Spain. Vet Parasitol. 2014;202:207-16.

18. Pires CA. Phlebotomus of Portugal. I-Natural infestation of Phlebotomus ariasi Tonnoir, 1921 and Phlebotomus perniciosus Newstead, 1911, by Leishmania in the zoonotic focus of Arrábida (Portugal). Ann Parasitol Hum Comp. 1984;59:521-4.

19. Cortes S, Afonso MO, Alves-Pires C, Campino L. Stray dogs and leishmaniasis in urban areas, Portugal. Emerg Infect Dis. 2007;13:1431-2.

20. Paltrinieri S, Solano-Gallego L, Fondati A, Lubas G, Gradoni L, Castagnaro $M$, et al. Guidelines for diagnosis and clinical classification of leishmaniasis in dogs. J Am Vet Med Assoc. 2010;236:1184-91.

21. Maia C, Coimbra M, Ramos C, Cristóvão J, Cardoso L, Campino L. Serological investigation of Leishmania infantum, Dirofilaria immitis and Angiostrongylus vasorum in dogs from southern Portugal. Parasites Vectors. 2015;8:152.

22. Maia C, Alwassouf S, Cristóvão JM, Ayhan N, Pereira A, Charrel RN, et al. Serological association between Leishmania infantum and sand fly fever Sicilian (but not Toscana) virus in sheltered dogs from southern Portugal. Parasites Vectors. 2017;10:92.

23. Alwassouf S, Maia C, Ayhan N, Coimbra M, Cristovao JM, Richet H, et al. Neutralization-based seroprevalence of Toscana virus and sandfly fever Sicilian virus in dogs and cats from Portugal. J Gen Virol. 2016;97:2816-23.

24. Maia C, Almeida B, Coimbra M, Fernandes M, Cristóvão J, Ramos C, et al. Bacterial and protozoal agents of canine vector-borne diseases in the blood of domestic and stray dogs from southern Portugal. Parasites Vectors. 2015:8:138.

25. Cruz I, Cañavate C, Rubio JM, Morales MA, Chicharro C, Laguna F, et al. A nested polymerase chain reaction ( $L n-P C R$ ) for diagnosing and monitoring Leishmania infantum infection in patients co-infected with human immunodeficiency virus. Trans R Soc Trop Med Hyg. 2002;96(Suppl. 1):S185-9.

26. Maia C, Nunes M, Cristóvão J, Campino L. Experimental canine leishmaniasis: clinical, parasitological and serological follow-up. Acta Trop. 2010;116:193-9.

27. Volf P, Volfova V. Establishment and maintenance of sand fly colonies. J Vector Ecol. 2011;36:S1-9.

28. Sergeant E, Perkins N. Epidemiology for field veterinarians: an introduction. Wallingford: CAB International; 2015.

29. Schober P, Boer C, Schwarte LA. Correlation coefficients. Anesth Analg. 2018;126:1763-8.

30. Miró G, Cardoso L, Pennisi MG, Oliva G, Baneth G. Canine leishmaniosisnew concepts and insights on an expanding zoonosis: part two. Trends Parasitol. 2008;24:371-7.

31. Solano-Gallego L, Koutinas A, Miró G, Cardoso L, Pennisi MG, Ferrer L, et al. Directions for the diagnosis, clinical staging, treatment and prevention of canine leishmaniosis. Vet Parasitol. 2009;165:1-18.

32. Foglia Manzillo V, Di Muccio T, Cappiello S, Scalone A, Paparcone R, Fiorentino $E$, et al. Prospective study on the incidence and progression of clinical signs in naïve dogs naturally infected by Leishmania infantum. PLoS Negl Trop Dis. 2013;7:e2225.

33. Iniesta L, Fernández-Barredo S, Bulle B, Gómez MT, Piarroux R, Gállego M et al. Diagnostic techniques to detect cryptic leishmaniasis in dogs. Clin Diagn Lab Immunol. 2002;9:1137-41.

34. Otranto D, Paradies P, de Caprariis D, Stanneck D, Testini G, Grimm F, et al. Toward diagnosing Leishmania infantum infection in asymptomatic dogs in an area where leishmaniasis is endemic. Clin Vaccine Immunol. 2009; 16:337-43.
35. Oliva G, Scalone A, Foglia Manzillo V, Gramiccia M, Pagano A, Di Muccio $\mathrm{T}$, et al. Incidence and time course of Leishmania infantum infections examined by parasitological, serologic, and nested-PCR techniques in a cohort of naive dogs exposed to three consecutive transmission seasons. J Clin Microbiol. 2006;44:1318-22.

36. Paradies P, Sasanelli M, de Caprariis D, Testini G, Traversa D, Lia RP, et al. Clinical and laboratory monitoring of dogs naturally infected by Leishmania infantum. Vet J. 2010;186:370-3.

37. Campino L, Maia C. The role of reservoirs: canine leishmaniasis. In: PonteSucre A, Diaz E, Padron-Nieves M, editors. Drug resistance in Leishmania parasites. Consequences, molecular mechanisms and possible treatments. 2nd ed. Viena: Springer; 2018. p. 59-83.

38. Cortes S, Vaz Y, Neves R, Maia C, Cardoso L, Campino L. Risk factors for canine leishmaniasis in an endemic Mediterranean region. Vet Parasitol. 2012;189:189-96.

39. Brianti E, Gaglio G, Napoli E, Falsone L, Prudente C, Solari Basano F, et al. Efficacy of a slow-release imidacloprid (10\%)/flumethrin (4.5\%) collar for the prevention of canine leishmaniosis. Parasites Vectors. 2014;7:327.

40. Strauss-Ayali D, Jaffe CL, Burshtain O, Gonen L, Baneth G. Polymerase chain reaction using noninvasively obtained samples, for the detection of Leishmania infantum DNA in dogs. J Infect Dis. 2004;189:1729-33.

41. Karakuş M, Töz S, Ertabaklar H, Paşa S, Atasoy A, Arserim SK, et al. Evaluation of conjunctival swab sampling in the diagnosis of canine leishmaniasis: a two-year follow-up study in Çukurova Plain, Turkey. Vet Parasitol. 2015;214:295-302.

42. Gaglio G, Napoli E, Arfuso F, Abbate JM, Giannetto S, Brianti E. Do different LED colours influence sand fly collection by light trap in the Mediterranean? Biomed Res Int. 2018;2018:6432637.

43. VIkova M, Rohousova I, Drahota J, Stanneck D, Kruedewagen EM, Mencke $\mathrm{N}$, et al. Canine antibody response to Phlebotomus perniciosus bites negatively correlates with the risk of Leishmania infantum transmission. PLoS Negl Trop Dis. 2011;5:e1344.

44. Pereira A, Cristóvão JM, Vilhena H, Martins Â, Cachola P, Henriques J, et al. Antibody response to Phlebotomus perniciosus saliva in cats naturally exposed to phlebotomine sand flies is positively associated with Leishmania infection. Parasites Vectors. 2019;12:128.

45. Souza AP, Andrade BB, Aquino D, Entringer P, Miranda JC, Alcantara R, et al. Using recombinant proteins from Lutzomyia longipalpis saliva to estimate human vector exposure in visceral leishmaniasis endemic areas. PLoS Negl Trop Dis. 2010;4:e649.

46. Quinnell RJ, Soremekun S, Bates PA, Rogers ME, Garcez LM, Courtenay O. Antibody response to sand fly saliva is a marker of transmission intensity but not disease progression in dogs naturally infected with Leishmania infantum. Parasites Vectors. 2018;11(1):7.

\section{Publisher's Note}

Springer Nature remains neutral with regard to jurisdictional claims in published maps and institutional affiliations.

\footnotetext{
Ready to submit your research? Choose BMC and benefit from:

- fast, convenient online submission

- thorough peer review by experienced researchers in your field

- rapid publication on acceptance

- support for research data, including large and complex data types

- gold Open Access which fosters wider collaboration and increased citations

- maximum visibility for your research: over $100 \mathrm{M}$ website views per year
}

At BMC, research is always in progress.

Learn more biomedcentral.com/submissions 